\title{
Mathematical modeling of sorption and desorption dynamics in adsorption systems \\ Natalia Sorokova $^{1}$, Julia Kolchyk ${ }^{2}$, Rodion Sorokovoi ${ }^{3}$
}

\begin{abstract}
1. National Technical University of Ukraine "KPI named after Igor Sikorsky", Kiev, 02000, Polytechnic Street, 6, E-mail: n.sorokova@ukr.net
\end{abstract}

2. Kyiv National University of Construction and Architecture, Kiev, 03680, Povitroflotsky Avenue, 31, E-mail: yulia@orblink.kiev.ua

3. Institute of Engineering Thermophysics of the National Academy of Sciences of Ukraine, Kiev, 03164, Bulakhovsky street, 2, E-mail: emo4ka69@gmail.com

A mathematical model and the results of calculating the dynamics of heat and mass transfer and phase transformations in the processes of sorption and desorption in solid porous sorbents in adsorption systems are presented. The calculation results indicate the adequacy of the mathematical model and the effectiveness of the calculation method.

Keywords - adsorption, desorption, adsorbent, adsorbate, mathematical modeling, intensity of phase transitions, mathematical modeling, dynamics of heat and mass transfer.

Heat and mass transfer processes of sorption and desorption are an integral part of many production technologies of energy, chemical, construction and other industries. Their passage in heterogeneous porous systems is accompanied by phase transitions and possible

deformation of the solid phase. The optimal organization of sorption and desorption ensures the preservation of the adsorption properties of sorbents and energy efficiency of production. Differences in the known mathematical models [1, 2] used to describe the dynamics of these processes are due to differences in the type and number of the main required functions and ways of closing the initial transfer equations. The methods of finding the intensity of phase transitions in them are quite approximate. Activation processes, the intensity of which significantly depends on temperature, have a significant effect on the dynamics of evaporation, condensation and diffusion transfer. The dynamics of sorption and desorption is characterized by the intensity of two competing processes: condensation of adsorbent molecules on the surface of the adsorbate layer covering the inner and outer surfaces of the porous body and evaporating molecules that are located near the free surface of the adsorbate layer and have reached the activation energy.

The intensity of the phase transition on the outer surface of the body is found by the formula [3]

$$
I=\gamma_{\mathrm{c}}\left\{\bar{\delta}(2-\bar{\delta})\left[\exp \left(A /\left.R T\right|_{\mathrm{v}=0}\right)-1\right]^{-1}-\varphi_{\mathrm{e} . \mathrm{m} .}\left[\exp \left(A / R T_{\mathrm{e} . \mathrm{m} .}\right)-1\right]^{-1}\right\}, \quad \gamma_{\mathrm{c}}=\varepsilon \rho_{\mathrm{fl}} \delta * / 4
$$

where $\varepsilon$ is the radiation coefficient; $\rho_{\mathrm{fl}}$ is the density of the liquid; $\delta^{*}$ is the thickness of the condensate layer in which the evaporation process takes place; the value $\bar{\delta}=\delta / \delta^{*}$ is found from the conditions: for $0<\delta<\delta^{*}$ and $\bar{\delta}=1$ with $\delta>\delta^{*}, \delta$ the thickness of the condensate layer; $A$ is the activation energy; $R$ is the universal gas constant; $\varphi_{\text {en }}$ relative humidity of the medium, $\varphi_{\mathrm{e} . \mathrm{m} .}=P_{\mathrm{v}} / P_{\mathrm{S}}, P_{\mathrm{v}}$ water vapor partial pressure, $P_{\mathrm{s}}$ saturation pressure; $T$ is temperature; $v$ is normal to the body surface. The first term in parentheses characterizes the intensity of evaporation of the adsorbate, and the second - the intensity of condensation of the sorbent. When the first term exceeds the second - the resultant process is the desorption of liquid, otherwise the adsorption of steam.

The intensity of phase transformations $I_{V}$ in a unit volume of a porous body is the product of the intensity of phase transition I on the liquid surface on the walls of partially filled 
capillaries and the total area $S$ of the contact surface of liquid and gas phases $I_{V}=I S$. To determine the function $\mathrm{S}$ obtained formula [3]

$$
S=\frac{2 \sqrt{1-\varphi_{\mathrm{b}}}}{\rho_{\mathrm{fl}} \delta^{*}} \frac{\partial U_{\mathrm{fl}}}{\partial \varphi_{\mathrm{b}}}, \quad\left(S_{\mathrm{min}} \leq S \leq S_{\mathrm{max}}\right),
$$

where $\varphi_{\mathrm{b}}$ is the adsorption humidity of the body, equal to the relative humidity of the gas, which according to the sorption-desorption isotherm corresponds to the volume concentration of the liquid $U_{\mathrm{fl}}$ at a given moment in time at a given point of the porous body; the derivative $\partial U_{\mathrm{fl}} / \partial \varphi_{\mathrm{b}}$ is found from the sorption isotherm equation.

Under the condition of local thermodynamic equilibrium, when $\left.T\right|_{\nu=0}=T_{\text {i.m. }}$, from (1) and (2) follows the expression for the intensity of phase transformations in the pores of the body

$$
I_{V}=\gamma_{\mathrm{c}} \frac{2 \sqrt{1-\varphi_{\mathrm{b}}}}{\rho_{\mathrm{fl}} \delta^{*}} \frac{\partial U_{\mathrm{fl}}}{\partial \varphi_{\mathrm{b}}}\left[\exp \left(\frac{A}{R T}\right)-1\right]^{-1}\left(\varphi_{\mathrm{b}}-\varphi\right),
$$

Where $\varphi=U_{\mathrm{v}} /\left[\Psi_{\mathrm{v}} P_{\mathrm{S}}(T)\right]$ is the relative humidity of the vapor-gas mixture in the capillaries $U_{\mathrm{v}}$ is volumetric concentrations of vapor phase; $\Psi_{\mathrm{v}}$ is volume fraction of the vapor in the porous body.

The thickness of the condensate layer is determined by the formula Nikitenko N.I. [4]

$$
\delta=\delta * \bar{\delta}=\delta *\left(1-\sqrt{1-P_{\mathrm{v}} / P_{\mathrm{eq}}}\right)=\delta *(1-\sqrt{1-\varphi}) .
$$

In accordance with (4), for given values of $T$ and $\varphi$ the thickness of the condensate layer on the walls of the pores is the same and equal to $\delta$ for capillaries of radius $r>\delta$, and the capillaries of radius $r \leq \delta$ are completely filled with liquid.

If the capillary-porous body is an adsorbent, in the process of sorption on its surface a monomolecular layer of condensate is formed, the molecules of which are most strongly bound to the adsorbent. Subsequent layers of bound liquid are held less tightly, and their properties approach the properties of the free liquid as they move away from the solid surface. To identify the nature and quantify the degree of influence of additional forces of interaction of a liquid particle with a solid body on the activation energy, it is convenient to use the potential of Sutherland, it describing the interaction of solid spheres with diameter $\sigma: \Phi(r)=\infty$ when $r \leq \sigma$ and $\Phi(r)=-B^{\prime} r^{-\gamma^{\prime}}$ when $r>\sigma$, where $\mathrm{B}^{\prime}$ and $\gamma^{\prime}$ are positive constants, $r$ is the distance between the spheres. Given the interaction of the liquid particle with the solid, the expression for the activation energy $A_{\text {ad }}$ can be written in the form $A_{a d}=A+\Delta A=A+B r^{-\gamma}$ where $\Delta A$ is the additional activation energy of the adsorbate molecules.

Wetting of the sorbent is carried out by supplying moist steam to its outer surface. The spent sorbent is dried by supplying it with energy from an external coolant.Transfer in capillaryporous bodies is carried out as a result of realization of several mechanisms: molecular (diffuse) and convective (filtration) transfer and transfer owing to phase transformations. In adsorption apparatuses, the processes of humidification and regeneration of sorbents are carried out at pressures lower than atmospheric. Therefore, the content of inert gases in the pores can be neglected. The movement of moisture in the material occurs in the form of liquid and vapor. Mathematical model of diffusion-filtration heat and mass transfer in a capillary-porous body, describing the processes of sorption and desorption includes the energy equation for the system as a whole and the equation of mass transfer of liquid and vapor

$$
\begin{gathered}
c_{\mathrm{ef}}\left(\partial T / \partial t+w_{\mathrm{ef}} \nabla T\right)=\nabla\left(\lambda_{\mathrm{ef}} \nabla T\right)-L I_{V}, \\
\partial U_{\mathrm{fl}} / \partial t+\nabla\left(w_{\mathrm{fl}} U_{\mathrm{fl}}\right)=\nabla\left[D_{\mathrm{fl}}\left(\nabla U_{\mathrm{fl}}+\delta_{\mathrm{fl}}^{T} \nabla T\right)\right]-I_{V},
\end{gathered}
$$




$$
\partial U_{\mathrm{v}} / \partial t+\nabla\left(w_{\mathrm{v}} U_{\mathrm{v}}\right)=\nabla\left[D_{\mathrm{v}}\left(\nabla U_{\mathrm{v}}+\delta_{\mathrm{v}}^{T} \nabla T\right)\right]+I_{V}
$$

Here $U_{\mathrm{fl}}, U_{\mathrm{v}}$ - volumetric concentrations of liquid and vapor phases; $T$ - temperature; $t$ - time; $c_{\text {ef }}-$ effective heat capacity, $c_{\mathrm{ef}}=c_{\mathrm{s}} \rho_{\mathrm{s}}(1-\Pi)+c_{\mathrm{fl}} U_{\mathrm{fl}}+c_{\mathrm{v}} U_{\mathrm{v}} ; \Pi-$ the porosity of the adsorbent; $\lambda_{\mathrm{ef}}-$ effective thermal conductivity, $\lambda_{\mathrm{ef}}=\lambda_{\mathrm{s}}(1-\Pi)+\lambda_{\mathrm{fl}} U_{\mathrm{fl}} / \rho_{\mathrm{fl}}+\lambda_{\mathrm{v}} U_{\mathrm{v}} / \rho_{\mathrm{v}} ; \nabla$ is the Hamilton operator; $w_{\mathrm{ef}}-$ effective filtration rate, $w_{\mathrm{ef}}=\left(w_{\mathrm{fl}} c_{\mathrm{fl}} U_{\mathrm{fl}}+w_{\mathrm{v}} c_{\mathrm{v}} U_{\mathrm{v}}\right) / c_{\mathrm{ef}} ; w_{\mathrm{fl}}, \mathrm{w}_{\mathrm{v}}-$ are the velocity vectors of the filtration motion of the liquid and vapor phases, which, according to the Darcy law $w_{\chi}=-K_{0} K_{\chi} / \eta_{\chi} \nabla P_{\chi}(\chi=\mathrm{fl}, \mathrm{v})$ are proportional to gradient the phases $\nabla P_{\mathrm{fl}}$ and $\nabla P_{\mathrm{v}} ; L$ is the heati of phase transformation; $I_{V}$ specific intensity of phase transformations in the pores of the body; $D_{\mathrm{fl}}, \quad D_{\mathrm{v}}-$ are the effective diffusion coefficients of the liquid and the vapor, $D_{\mathrm{fl}}=\gamma_{D}\left[\exp \left(A_{D} / R T\right)-1\right]^{-1}$ (the formula is Nikitenko N.I. [4]), $D_{\mathrm{v}}=\gamma_{\mathrm{v}} T^{3 / 2} / P_{\mathrm{v}}$ (from the kinetic theory of gases), here $A_{D}$ is the activation energy of the liquid molecules for the diffusion process; $\gamma_{D}, \gamma_{\mathrm{v}}=$ const.

The pressures $P_{\mathrm{fl}}$ and $P_{\mathrm{v}}$ are calculated through the functions $U_{\mathrm{fl}}, U_{\mathrm{v}}$ and $T$ according to the following algorithm: volume fractions of the skeleton $\Psi_{\mathrm{s}}$, liquid $\Psi_{\mathrm{fl}}$ and vapor $\Psi_{\mathrm{v}}$ in the porous body: $\Psi_{\mathrm{s}}=1-\Pi, \Psi_{\mathrm{fl}}=U_{\mathrm{fl}} / \rho_{\mathrm{fl}}, \Psi_{\mathrm{v}}=1-\Psi_{\mathrm{s}}-\Psi_{\mathrm{fl}}$, partial densitie and pressure of vapor $\rho_{\mathrm{v}}=U_{\mathrm{v}} / \Psi_{\mathrm{g}}$, $P_{\mathrm{\Pi}}=\rho_{\mathrm{\Pi l}} R_{\mathrm{y}} T / \mu_{\Pi}$. The pressure of liquid phase: $P_{\mathrm{fl}}=P_{\mathrm{g}}+P_{\mathrm{c}}$, where capillary pressure $P_{\mathrm{c}}$ [5]

$$
P_{\mathrm{c}}=2 \sigma(T) \int_{r_{\min }}^{r_{\max }} \frac{\theta(r)}{r} f(r) d r / \int_{r_{\min }}^{r_{\max }} \theta(r) f(r) d r=\frac{2 \sigma(T)}{r^{*}}, \quad r_{\min }<r^{*}<r_{\max } .
$$

Here, $\theta(r)$ is the volume fraction of the capillary filled with liquid; $f(r)$ is the differential function of pore size distribution; $\sigma(T)$ is the coefficient of surface tension; $r^{*}$ is the characteristic parameter of the dispersion of pore sizes; $r_{\min }$ and $r_{\max }$ are the minimum and maximum pore radii in a unit volume.

The boundary conditions of the heat-mass transfer of the third kind for the processes of wetting and drying of the sorbents are presented as follows:

$$
\begin{gathered}
\left.\lambda_{\mathrm{ef}} \frac{\partial T}{\partial v}\right|_{v=0}=\alpha\left(T_{\mathrm{e} . \mathrm{m} .}-\left.T\right|_{v=0}\right)-L I,(9) ; \quad D_{\mathrm{fl}}\left(\left.\frac{\partial U_{\mathrm{fl}}}{\partial v}\right|_{v=0}+\left.\delta_{\mathrm{fl}}^{T} \frac{\partial T}{\partial v}\right|_{\nu=0}\right)+\left.\nabla\left(w_{\mathrm{fl}} U_{\mathrm{fl}}\right)\right|_{\nu=0}= \pm I,(10) ; \\
D_{\mathrm{v}}\left(\left.\frac{\partial U_{\mathrm{v}}}{\partial v}\right|_{v=0}+\left.\delta_{\mathrm{v}}^{T} \frac{\partial T}{\partial v}\right|_{v=0}\right)+\left.\nabla\left(w_{\mathrm{v}} U_{\mathrm{v}}\right)\right|_{v=0}=\gamma_{\mathrm{v} \text { e.m. }}\left(\left.U_{\mathrm{v}}\right|_{\nu=0} \mp \rho_{\mathrm{v} \text { e.m. } .} \psi_{\mathrm{v}}\right) .
\end{gathered}
$$

The process of sorbents regeneration in sorption apparatuses often occurs during conductive energy supply. At the interface of the heat exchange surface with the adsorbent, the conditions of the fourth kind are set

$$
\left.\lambda \frac{\partial T}{\partial v}\right|_{\nu=-0}=\lambda \text { ef }\left.\frac{\partial T}{\partial v}\right|_{v=+0} .
$$

The solution of the system (3) -- (6) under certain boundary conditions (9) -- (12) makes it possible to determine the values at each point of the body at each instant of time of temperature, volumetric concentrations of the liquid, vapor and air phases and other parameters, as well as the completion time of adsorption or desorption processes. For this, a grid method has been developed, which is based on an explicit three-layer recalculating difference scheme Nikitenko N.I. [5] and a computer program for the calculation has been compiled.

The results of dynamics of moisture content and temperature change in a layer of silicagel during its moistened and regenerationare shown in Fig. 1,2. 

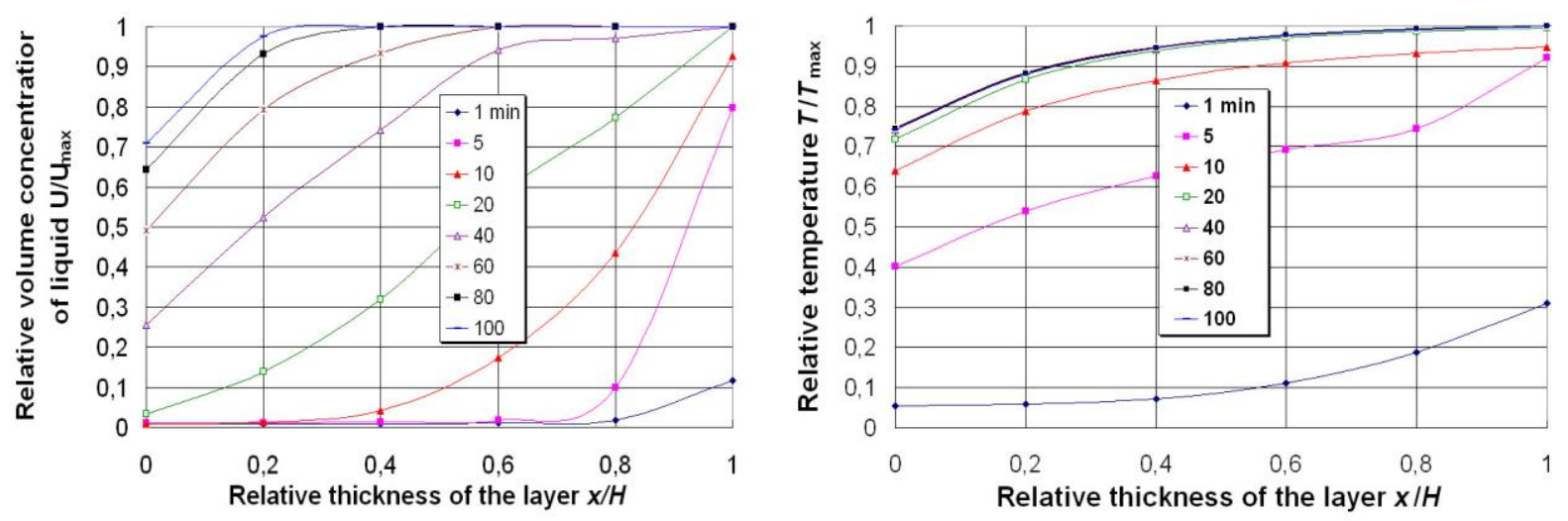

Fig. 1 The relative volumetric concentration of the liquid $\bar{U}_{\mathrm{fl}}=U_{\mathrm{fl}}(x) / U_{\mathrm{eq}}$ and the temperature $\bar{T}(x)=T(x) / T_{\max }$ in the silicagel layer at different times during adsorption. Parameters of vapor medium: $\varphi_{\mathrm{en}}=100 \%, T_{\mathrm{en}}=10{ }^{\circ} \mathrm{C}$, body parameters: $T_{0}=10{ }^{\circ} \mathrm{C}, T_{\text {мax }}=195{ }^{\circ} \mathrm{C}$.
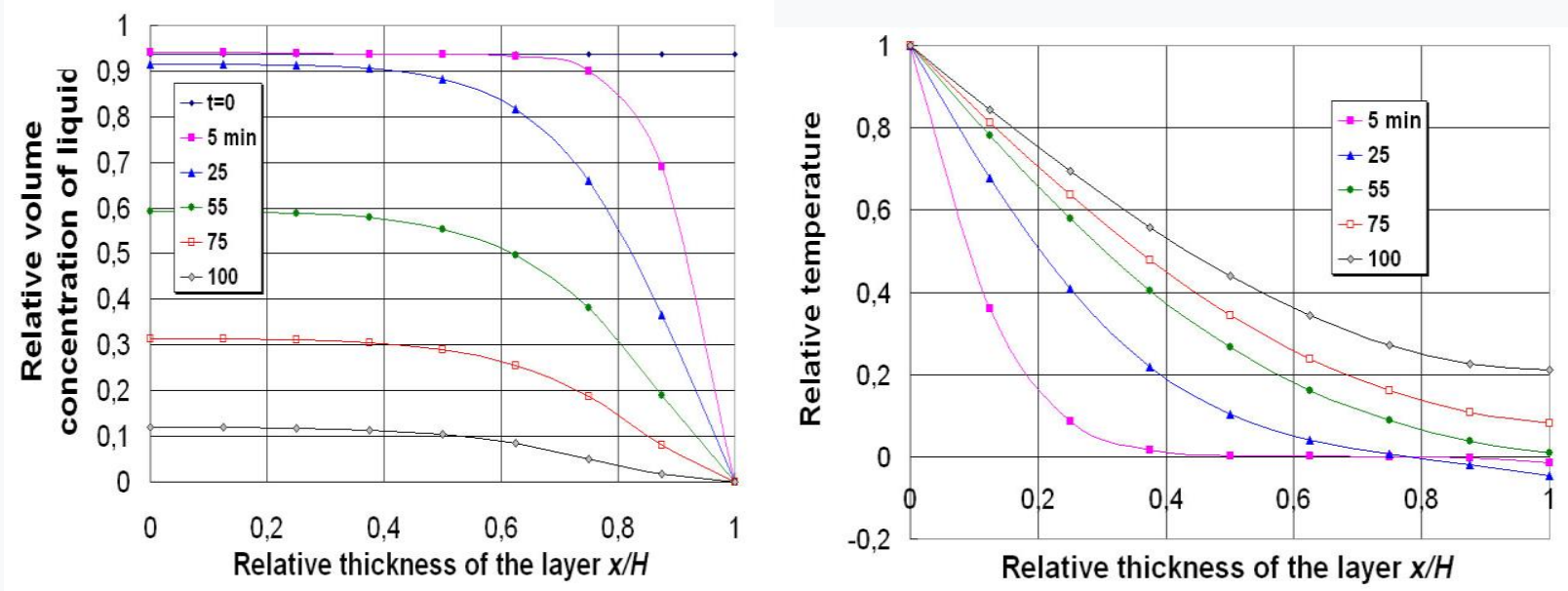

Fig.2 Relative values of volumetric liquid concentration $\bar{U}_{\mathrm{fl}}=\left(U_{\mathrm{fl}}(x)-U_{\mathrm{eq}}\right) / U_{\mathrm{fl} 0}$ and the temperature $\bar{T}(x)=\left(T(x)-T_{0}\right) /\left(T_{\text {h.s. }}-T_{0}\right)$ of the silicagel at different times of drying with a conductive method of supplying heat. $T_{0}=20^{\circ} \mathrm{C}, T_{\mathrm{h} . \mathrm{s}}=150^{\circ} \mathrm{C}$.

\section{Conclusion}

The results of comparing the calculated and experimental data on the kinetics of humidification and regeneration of different sorbents [5] indicate the adequacy of the mathematical model and the effectiveness of the calculation method.

\section{References}

1. Keltsev NV Fundamentals of adsorption technology. Monograph (2 th ed.). M $\therefore$ Khimiya. 590 p. URL https://www.twirpx.com/file/1054811/. (Book)

2 Zhang L. Z. (2000). A three- dimensional non-equlibrium model for an intermittent adsorption cooling system. Solar energy. Vol. 69, № 1. P. 27-35. . http://dx.doi.org/ 10.1016/S0038-092X(00)00010-4.

3 Nikitenko N.I., Snezhkin Yu.F., Sorokovaya N.N. (2010) Development of the theory andmethods for calculating the dynamics of sorption and desorption. Journal of Engineering Physics and Thermophysics. V. 83, № 4. P. 832-842. DOI: 10.1007/s10891-010-0399-y

4. Nikitenko N.I. ( 2002) Investigation of the Dynamics of Evaporation of Condensed Bodies on the Basis of the Law of Spectral-Radiation Intensity of Particles. Journal of Engineering Physics and Thermophysics. V.75, № 3, P.684-692 . https://doi.org/10.1023/A:1016813711896

5. Nikitenko N.I., Snezhkin Yu.F., Sorokovaya N.N., Kolchik Yu.N. (2014) Molecular radiation theory and methods for calculating heat and mass transfer. Kiev: Naukova Dumka, 744 p. http://ittf.kiev.ua/wp-content/uploads/2016/12/nikitenko.pdf (Book). 